\section{Paternal and maternal transi- tion to parenthood: the risk of postpartum depression and parenting stress}

\author{
Maria Stella Epifanio, Vitalba Genna, \\ Caterina De Luca, Michele Roccella, \\ Sabina La Grutta \\ Department of Psychological and \\ Educational Sciences, University of \\ Palermo, Italy
}

\section{Abstract}

Transition to parenthood represents an important life event increasing vulnerability to psychological disorders. Postpartum depression and parenting distress are the most common psychological disturbances and a growing scientific evidence suggests that both mothers and fathers are involved in this developmental crisis. This paper aims to explore maternal and paternal experience of transition to parenthood in terms of parenting distress and risk of postpartum depression. Seventy-five couples of first-time parents were invited to compile the Edinburgh Postnatal Depression Scale and the Parenting Stress Index-Short Form in the first month of children life. Study sample reported very high levels of parenting distress and a risk of postpartum depression in $20.8 \%$ of mothers and $5.7 \%$ of fathers. No significant correlation between parenting distress and the risk of postpartum depression emerged, both in mothers than in fathers group while maternal distress levels are related to paternal one. The first month after partum represents a critical phase of parents life and it could be considered a developmental crisis characterized by anxiety, stress and mood alterations that could have important repercussions on the child psycho-physical development.

\section{Introduction}

Transition to parenthood is a psychological and developmental complex process characterized by several personal and family changes requiring adjustment. The birth of a baby can result in profound changes to lifestyle and recreation, sleep patterns, couple relationships and identity. 1

Both of parents have to cope with a identity and family transformation; the parenting function development, that is different in men and in women, usually results in the acquisition of a good parenting competence. Sometimes, this process leads to psychological disturbances or psychopathological status like post partum depression (PPD) that has recently been studied both for increasing prevalence and for its effects on child development and health. ${ }^{2}$

There has been no univocal evidence regarding the classification of PPD: some authors suggest that PPD is not a specific disturbance and that can be assimilated to major depressive disorder; other authors evidence that PPD is a specific mood alteration related to maternity. According to DSM-5, 3 PPD is a mood episode that could have its onset either during pregnancy or postpartum; between 3\% and $6 \%$ of women experience the onset of a major depressive episode during pregnancy or in the weeks or months following delivery and $50 \%$ of postpartum major depressive episodes begin prior to delivery. ${ }^{3}$ Although there is no general agreement about the time of PPD assessment and other literature evidences affirm that maternal vulnerability to PPD could extent to the first year after the birth. ${ }^{4}$

Epidemiological studies have found that in developed countries $10-15 \% t$ of first-time mothers were affected by postpartum depression and they have focused on predictors on it. ${ }^{5}$ Scientific literature identified a complex etiology of PPD because there are a lot of predictive factors such as history of depression during pregnancy and before pregnancy, life events, absence or paucity of social support, quality marital relationship and low socioeconomic status. ${ }^{6}$ Postpartum depression is characterized by persistent sadness, low esteem, anxiety, irritability and sleep/appetite alterations, dysphoria, loneliness, emotional lability, insomnia, confusion, guilt, and suicidal ideation. ${ }^{7}$ These symptoms are disabling and enabling to cope with normal developmental tasks in the child care and in the care for themselves.

For this symptomatological heterogeneity, the assessment of psychological disturbances in the postpartum period depends on factors such as time of assessment, definition of depression, instrument used to measure depression, and the cultural characteristics of the population studied. ${ }^{8}$

For many years, research studies have suggested that antenatal and postnatal depression regards mainly maternal figure, since the mother represents the most important caregiver in the first life time of a baby. Recently, researchers have shown an increased interest in paternal psychological status because it is strictly related to maternal one and to child development. ${ }^{9}$ These few studies evidence that also fathers can be affected by this type of mood alterations and that the transition to parenthood represents a stressor and an anxiety experience for $10 \%$ of fathers, 10 revaluating the paternal role in family functioning after childbirth. $^{9-11}$

Scientific findings about the rate of diag-
Correspondence: Maria Stella Epifanio, Department of Psychological and Educational Sciences, University of Palermo, via delle Scienze, 90128 Palermo, Italy.

Tel.: +39.091.238.97714 - Fax: +39.091.651.3825.

E-mail: mariastella.epifanio@unipa.it

Key words: Transition to parenthood; parenting distress; postpartum depression.

Acknowledgments: we are grateful to all parents and pediatricians who took part in the research project.

Contributions: the authors contributed equally.

Conflict of interest: the authors declare no potential conflict of interest.

Received for publication: 13 February 2015.

Accepted for publication: 25 May 2015.

This work is licensed under a Creative Commons Attribution NonCommercial 3.0 License (CC BYNC 3.0).

(C) Copyright M.S. Epifanio et al., 2015

Licensee PAGEPress, Italy

Pediatric Reports 2015; 7:5872

doi:10.4081/pr.2015.5872

nosed paternal PPD are divergent: a relatively recent meta-analysis reported that a range between $2 \%$ and $25 \%$ of first-time fathers suffers PPD symptoms; ${ }^{12}$ in the cases of concomitant partner PPD, the rate of paternal PPD increases at $24-50 \%$. Divergence and approximation of these estimations reflect the complex nature of paternal depression, its difference from maternal one, and methodological measurement difficulties. ${ }^{13}$

Indeed, the postpartum distress manifestation is different between mothers and fathers, principal paternal PPD symptoms, unlike female clinical picture, are angers attacks, affective rigidity, self-criticism, exhaustion, alcohol and drug abuse. ${ }^{14}$ Men can present also somatic symptoms like indigestion, increased or decreased appetite, weight gain, diarrhea or constipation, headache, toothache, nausea and insomnia.13 Furthermore, the paternal PPD could begin over the first year postpartum, later than maternal one. ${ }^{8}$

The main paternal PPD predictors are the personal history of depression, the quality and functioning of marital relationship and above all the partner depression. ${ }^{12}$ It is known that when a mother is severely depressed, the risk of paternal depression redoubles. 15 On the other hand, Grussu and Quatraro have suggested that fathers could increase the risk of maternal depression, 16 if they are depressed or absents; and they could represent a protective factor for maternal depression, if they are 
healthy and supportive. The relationship between paternal PPD and maternal one is more investigated but the nature or the direction of it is not clear. It has been reported that maternal PPD is a predictor of paternal one since the first is higher during the three months postpartum. ${ }^{8}$ Based on the existing knowledge of maternal PPD, literature suggests that also paternal PPD could be related with hormonal changes regarding alteration of testosterone, estrogen, vasopressin, prolactin and cortisol levels. ${ }^{10}$ In addition to mood disturbances, high parenting distress levels could also be considered a important factor compromising the parenting competence and the daily child care. 17 Parenting stress is a construct related to the parent role and influenced by expectations and perceptions of child characteristics, parent characteristics and parentalinfant interaction quality. It is the constellation of bio-psycho-social factors (anxiety, uncomfortable, psychological and emotional tension, negative/maladaptive coping to stressful events, emotional incompetence, etc.) which distorts the adaptive reaction to stressors and predispose the subject to high psychological and socio-relational vulnerability. 18,19

Woman is the principal caregiver and a good mother have to be loving and able to satisfy her tasks in the care of children. In this way, to admit that a woman become mother could have the need of spaces (such as work, friendship and hobbies) different from parenting ones is very difficult; mothers suffer specific consequences such as increased household chores, continuous availability, lack of sleep and leisure to dedicate to themselves and feeling of being trapped and feeling of carelessness. ${ }^{20}$ Furthermore, working women have to renounce their work or have to reduce working hours, with consequences of remuneration and career prospects. ${ }^{21}$

The relationship between parenting stress and PPD emerged in several studies, ${ }^{22}$ evidencing the influence of individual and family variables on quality of early affective relations. ${ }^{17,23}$

In these way, studying the postpartum distress is very useful not only to expand the knowledge about depressive psychopathology but also to explore and prevent long-term negative effects on children's health.

Indeed, much scientific attention has been paid to the effect of maternal depressive status on early interaction mother-child compromising social, emotional, cognitive and physical development of children. ${ }^{24}$

Neurobiological studies have shown that a relationship with a depressive mother is able to provoke alteration in child development and to increase vulnerability for later psychopathological risk. ${ }^{25,26} \mathrm{~A}$ depressive mother is less empathic, less careful to child needs, less responsive, 12 she shows a sensitivity that is flat, not-synchronized to her child and poorly stimulating. The interaction between a child and his depressive mothers could be pathologically intrusive or detached.27,28 These situations compromise the maternal competence in the daily child management (put correctly the child to the bed, to use of seatbelt in the car or control the temperature in the bathe) and in the other important activities such as read, sing 0 tell a story. 29,30

This condition could have serious repercussions on child development, ${ }^{31}$ that vary on the base of the child age: difficult and inconsolable temperament, neurophysiologic alteration (colic, appetite/sleep symptoms) ${ }^{32}$ and delay of cognitive and/or motor functions in newborn; 33 affect disorders, compromised symbolic play, poor social ability, serious allergy or asthma in 12-24 months old children could emerge. ${ }^{34}$ During preschool age, low Q.I., sleep and psychosomatic disturbances could appear, ${ }^{35,36}$ while in scholar age low self-esteem, depressive or anxiety symptoms, intellective or attentive difficulties, reading or language disturbances, ${ }^{37}$ poor social ability, poor scholar profit could emerge. ${ }^{38,39}$ In adolescence, depressive, anxiety and attentive disturbances could be frequent. ${ }^{35,36}$ Although, the relation between maternal depressive status and compromised development child in not sure because the child is an active partner in this relation and could play an important role in this process.

Regarding these topics, this paper aims to: i) explore the maternal and paternal experience of transition to parenthood, in terms of parenting distress and PPD risk; ii) investigate the relationship between parenting distress and risk of PPD, in mothers and fathers; iii) evaluate the relationship between maternal and paternal psychological distress, in terms of parenting stress and PPD levels.

\section{Materials and Methods}

A cross-sectional study design was chosen to achieve mentioned aims. Study sample was composed by 75 couples of primiparae parents recruited in private pediatric ambulatories in Palermo and Trapani (Italy) during the first pediatric visit in the first month of children life (age $\mathrm{M}=22.38$ days; $\mathrm{SD}=6.5$ days). The time of assessment was chosen to evaluate psychological experience when first-time parents return at home and they meet child requests. In this period the mother get her mastery and responsibility back, she tends to recognize physical and psychological baby needs and she tends to establish dyadic reciprocity. Furthermore, the couple tends to build a new equilibrium and to get marital relations back.

Compiling mothers (age $\mathrm{M}=30.35$; $\mathrm{DS}=5.6$ ) and fathers (age $\mathrm{M}=34.45 ; \mathrm{DS}=5.2$ ) are
Italian, have medium-high scholar education (women: $\mathrm{M}=13.31 ; \mathrm{DS}=3$; men: $\mathrm{M}=12.71$; $\mathrm{DS}=1.5)$, they are married and live together, only the $61 \%$ of women and the $62 \%$ of men have a job. Study participants had natural childbirth and there are no complications in the pregnancy or delivery. Mothers and fathers separately compiled the Edinburgh Postnatal Depression Scale (EPDS) and the Parenting Stress Index-Short Form (PSI-SF).18,19,40,41 Informed consent was obtained from each participant before beginning the assessment during explanation of study purpose. After scoring and transforming in percentiles ones (according to Italian validations), descriptive statistics, correlation with $r$ Pearson and Chi Square test were conducted on validity protocols through Statistical Package for Social Science (SPSS-17). Some analyses were conducted separately for mothers and fathers, others on the couple of parents.

\section{Edinburgh Postnatal Depression scale}

There are several specific instruments to assess the risk of postpartum depression but the clinical differences between paternal and maternal postpartum depression make necessary to use a scale suitable for both manifestations. For these reasons, we chosen the Edinburgh Postnatal Depression Scale (EPDS), a self report composed by 10 items (eight addressing depressive symptoms and two inquiring about anxiety symptoms). The scale allows to identify the risk of clinical depression and the subclinical one, both in mothers and in fathers using different cut-off scores. According to Italian validation, ${ }^{41,42}$ in mothers sample cut off score is 9: scores lower than 9 report no depression risk or subclinical symptoms presence, scores higher than 9 mean risk of clinical PPD. For fathers, a cut-off score of $5 / 6$ was used to distinguish the absence of PPD risk from subclinical PPD symptoms while scores higher than 12 indicate risk of clinical PPD. In this study, the Cronbach's alpha reliability coefficient of this measure is 0.64 for mothers and 0.6 for fathers.

\section{The Parenting Stress Index short form}

It is the short version of the Parent Stress Index, an instrument greatly used to value the parenting stress that is defined by the Authors like the constellation of biological, psychological and social factors distorting the adaptive reaction to parental stressors and predispose the parent to psychological vulnerability. This self-report uses a three factors model to measure parenting stress, to which correspond the three subscales reported below. The scale of parenting distress (PD) defines the level of dis- 
tress which a parent perceives in his parenting role, linked to personal factors directly involved in this role. The scale of the dysfunctional interaction parent-child (P-CDI) values the parenting perception of a child that doesn't respond to the family expectations and of an interaction neither reinforcing nor rewarding with the child. The scale of the difficult child (DC) values how much the parent perceives his child as easy/difficult to manage, considering some of his behavioral characteristics. Each subscales scores are transformed in percentiles scores that are considered critical if overcome $85 \%$. The total score, obtained by the sum of the scores of the 3 subscales, can be interpreted as a stress index related to the only parenting role. The test includes also a Defensive Responding scale, useful to control the validity of the protocol, which indicates if the parent tends to give a better self-image, minimizing the problems and the perceived stress in the relationship with the child. In this study, the Cronbach's alpha reliability coefficient of this measure is 0.91 for mothers and 0.88 for fathers.

\section{Results}

The initial sample consisted of 150 participants (75 couples) of whom 44 (22 couples) reported in the PSI-SF Defensive Responding scale scores lower than 10 , cut-off score establishing protocol validity. These 22 couples were excluded from statistical analysis that were conducted on 53 couples (106 participants).

Concerning mothers, as measured by EPDS, $20,8 \%$ of mothers $(\mathrm{N}=11)$ reported high risk of postpartum depression and, as measured by PSI-SF, 98,1\% of them overcome critical cut-off in questionnaire global score (Table 1).
Indeed, mothers reported very high scores both in global distress level and in distress subscales (Table 2).

In mothers sample, correlation analysis with

r Pearson evidenced no significant correlations between PSI-SF domains scores and EPDS ones (Table 3).

Concerning fathers, as measured by EPDS,

Table 1. Prevalence of postpartum depression risk and parenting distress levels $(\mathrm{N}=53$ couples; $\mathrm{N}=106$ individuals).

\begin{tabular}{|c|c|c|c|}
\hline & Mothers, n (\%) & Fathers, n (\%) & Total, n (\%) \\
\hline $\begin{array}{l}\text { EPDS } \\
\text { No risk of PPD } \\
\text { Risk of subclinical PPD } \\
\text { Risk of clinical PPD }\end{array}$ & $\begin{array}{c}42(79.2) \\
- \\
11(20.8)\end{array}$ & $\begin{array}{c}40(75.5) \\
10(18.9) \\
3(5.7)\end{array}$ & $\begin{array}{l}82(77.4) \\
10(18.9) \\
14(13.2)\end{array}$ \\
\hline $\begin{array}{l}\text { Global PSI-SF } \\
\quad<85 \text { percentile } \\
>85 \text { percentile }\end{array}$ & $\begin{array}{c}1(1.9) \\
52(98.1)\end{array}$ & $\begin{array}{c}1(1.9) \\
52(98.1)\end{array}$ & $\begin{array}{c}2(1.9) \\
104(98.1)\end{array}$ \\
\hline $\begin{array}{l}\text { PD } \\
\quad<85 \text { percentile } \\
>85 \text { percentile }\end{array}$ & $\begin{array}{l}11(20.8) \\
42(79.2)\end{array}$ & $\begin{array}{l}8(15.1) \\
45(84.9)\end{array}$ & $\begin{array}{l}19(17.9) \\
87(82.1)\end{array}$ \\
\hline $\begin{array}{l}\text { P-CDI } \\
\quad<85 \text { percentile } \\
\quad>85 \text { percentile }\end{array}$ & $\begin{array}{c}6(11.3) \\
47(88.7)\end{array}$ & $\begin{array}{c}5(9.4) \\
48(90.6)\end{array}$ & $\begin{array}{l}11(10.4) \\
95(89.6)\end{array}$ \\
\hline $\begin{array}{l}\text { DC } \\
<85 \text { percentile } \\
>85 \text { percentile } \\
\end{array}$ & $\begin{array}{c}1(1.9) \\
52(98.1)\end{array}$ & $\begin{array}{c}1(1.9) \\
52(98.1)\end{array}$ & $\begin{array}{c}2(1.9) \\
104(98.1)\end{array}$ \\
\hline
\end{tabular}

EPDS, Edinburgh Postnatal Depression Scale; PPD, postpartum depression; PSI-SF, Parenting Stress Index-Short Form; PD, parenting distress; P-CDI, dysfunctional interaction parent-child; DC; difficult child.

Table 2. Descriptive statistics on Parenting Stress Index-Short Form scores ( $\mathrm{N}=53$ couples).

\begin{tabular}{lcccc} 
& Min. & Max. & Mean & SD \\
Mothers & & & & \\
PD & 19 & 53 & 42.00 & 9.977 \\
P-CDI & 13 & 55 & 39.85 & 10.352 \\
DC & 17 & 57 & 43.11 & 7.345 \\
Global PSI-SF & 56 & 150 & 124.96 & 21.815 \\
Fathers & & & & \\
PD & 20 & 53 & 41.94 & 8.236 \\
P-CDI & 17 & 54 & 40.36 & 8.200 \\
DC & 25 & 56 & 44.25 & 7.272 \\
Global PSI-SF & 74 & 145 & 126.55 & 18.421 \\
\hline
\end{tabular}

SD, standard deviation; PD, parenting distress; P-CDI, dysfunctional interaction parent-child; DC; difficult child; PSI-SF, Parenting Stress Index-Short Form.

Table 3 Correlations between Edinburgh Postnatal Depression Scale and Parenting Stress Index scores in mothers and fathers sample ( $\mathrm{N}=53$ couples).

\begin{tabular}{|c|c|c|c|c|c|c|c|c|c|c|}
\hline & $\begin{array}{l}\text { Maternal } \\
\text { EPDS }\end{array}$ & $\begin{array}{l}\text { Paternal } \\
\text { EPDS }\end{array}$ & $\begin{array}{c}\text { Maternal } \\
\text { PD }\end{array}$ & $\begin{array}{l}\text { Paternal } \\
\text { PD }\end{array}$ & $\begin{array}{l}\text { Maternal } \\
\text { PCDI }\end{array}$ & $\begin{array}{c}\text { Paternal } \\
\text { PCDI }\end{array}$ & $\begin{array}{l}\text { Maternal } \\
\text { DC }\end{array}$ & $\begin{array}{l}\text { Paternal } \\
\text { DCglobal }\end{array}$ & $\begin{array}{l}\text { Maternal } \\
\text { PSI-SFglobal }\end{array}$ & $\begin{array}{l}\text { Paternal } \\
\text { I PSI-SF }\end{array}$ \\
\hline Maternal EPDS & 1 & -0.108 & -0.254 & -0.162 & -0.042 & 0.126 & -0.257 & -0.082 & -0.223 & -0.048 \\
\hline Paternal EPDS & & 1 & 0.248 & -0.114 & 0.205 & -0.052 & 0.201 & -0.124 & $0.279 a$ & -0.123 \\
\hline Maternal PD & & & 1 & $0.534^{b}$ & $0.754^{b}$ & $0.427 \mathrm{~b}$ & 0.256 & 0.167 & $0.901^{b}$ & $0.495^{b}$ \\
\hline Paternal PD & & & & 1 & $0.484 \mathrm{~b}$ & $0.605^{b}$ & 0.221 & $0.346 \mathrm{a}$ & $0.548^{b}$ & $0.853^{\mathrm{b}}$ \\
\hline Maternal PCDI & & & & & 1 & $0.522^{b}$ & 0.145 & -0.013 & $0.868 \mathrm{~b}$ & $0.443^{b}$ \\
\hline Paternal PCDI & & & & & & 1 & -0.173 & 0.236 & $0.385^{b}$ & $0.809 \mathrm{~b}$ \\
\hline Maternal DC & & & & & & & 1 & $0.318 \mathrm{a}$ & $0.522^{b}$ & 0.147 \\
\hline Paternal DC & & & & & & & & 1 & 0.177 & $0.655^{b}$ \\
\hline Maternal Global & I-SF & & & & & & & & 1 & $0.486^{b}$ \\
\hline Paternal Global & & & & & & & & & & 1 \\
\hline
\end{tabular}

EPDS, Edinburgh Postnatal Depression Scale; PD, parenting distress; P-CDI, dysfunctional interaction parent-child; DC; difficult child; PSI-SF, Parenting Stress Index-Short Form. Different letters in the same column/row indicate different statistical significance. 
$5,7 \%$ of fathers $(\mathrm{N}=3)$ reported high risk of postpartum depression and $18.9 \%$ of them $(\mathrm{N}=10)$ reported borderline scores indicating risk of subclinical postpartum depression (Table 1). At PSI-SF (Table 2), like the mothers, 52 fathers (98.1\%) overcome critical cut-off in PSISF global score (Table 1). Indeed, fathers reported very high scores both in global distress level and in distress subscales (Table 2).

In fathers sample, correlation analysis with r Pearson (Table 3) evidenced no significant correlations between PSI-SF domains scores and EPDS ones.

Chi square also suggested that maternal risk of postpartum depression is significantly higher than paternal one $\left[\chi^{2}(2)=14.6\right.$, $\mathrm{P}=0.001$ ] while there is no significant differences between mothers and fathers about parenting distress $(\mathrm{P}>0.05)$.

To examine the relationship between maternal and paternal parenting experience in terms of psychological distress and postpartum depression, correlation analysis with $r$ Pearson were conducted (Table 3 ). There is no association between paternal and maternal post partum depression risk but there is an high association between maternal and paternal distress $(\mathrm{r}=0.486 ; \mathrm{P}<0.01)$ (Table 3$)$.

Additional analysis regarding the association between the desire of pregnancy and the level of post partum depression suggests that there is a significant difference between fathers who desired a child e fathers who did not desire a child in terms of post partum depression $\left[\chi^{2}(2)=6.1, P=0.045\right]$. Indeed, the absence of a child desire is more related with post partum depression in fathers but not in the mothers sample (Table 4).

Finally, to study the relation between breastfeeding and risk of maternal post partum depression, our results evidence a significant difference between mothers who breastfed their child and mothers who not breastfeed him in terms of post partum depression $\left[\chi^{2}(1)=3.8, \mathrm{P}=0.049\right]$ (Table 5).

\section{Discussion}

The presence of invalid protocols at PSI-SF Defensive Responding Scale evidenced probably social desirability bias. Parents could tend to give a better self-image, showing the fear to express sincerely their experience, minimizing the problems and the perceived stress in the relationship with the child.

Although study sample was composed by families without special needs, participants reported high levels of parenting distress.

Parenting distress means the level of distress that a parent is experiencing in his/ her new role, the rewarding nature of the interaction with the child, the parental perceptions of the child care. According to the literature, the parenting characterized by high distress level could be considered as a predictor factor of mood disturbance but in our sample high distress levels could be considered such a adaptive condition in the first weeks of child life, as demonstrated by the absence of association between PPD and parenting distress.

Transition to parenthood could be considered such as a developmental crisis in the couple history, first-time parents could lose individual and couple balance in the adjustment process to a baby and to his need, especially in the first month after partum, since the family routine is interrupted. Acquired relationship ways became unsuitable and new priorities and responsibility emerge.

During postpartum period, paternal parenting stress could be complicated by paternal perception of differences between gender roles: the father usually lives the economical pressure that could obstruct the paternal involvement in the parenting.

We believe that a limited length of parenting distress has an adaptive function, although a chronic length of parenting distress could became in psychopathological disturbances, especially if it is associated with risk factors like social support absence, reduced marital satisfaction and difficult child. In our sample the significant correlation between maternal and paternal parenting distress represents a risk condition that could compromise and drain the parenting coping with the daily child care.

The intrinsic complexity of the transition to parenthood could lead to constructive routes of development as well as regressive routes char- acterized by critical and problematic elements up to psychopatological conditions.

The high risk of PPD in the $20 \%$ of mothers sample evidences that the transition to parenthood is a very vulnerable period for women and principal risk factors are psychological, social and cultural one (OMS). ${ }^{43}$

In this way, the meaning of maternal PPD must trace the origin of the women' history and her psychological functioning.

The maternity is a psychological status: 4 becoming mother needs a profound reorganization of internal and external reality. The acquisition of maternal role derives from a profound elaboration process that allows the creation of a new mental arrangement named motherhood constellation by Stern. 44

This psychological arrangement starts to organize during the pregnancy and allows the maternal psychological reorganization through the definition of priority, values, interests and the development of affective harmony and affect mirroring with the child.

The change needs a global process of individual and family arrangement: the mother, consciously and unconsciously, elaborates her experience of daughter and the quality of relationship with her mother, she attempts to link fantasies about pregnancy, delivery and child.

These situation could contribute to threaten psychological health of mothers, who start to perceive a sense of inadequacy and loss of faith in their own abilities, reduced mood, agitation, anxiety, and significant loss of interest in routine activities, appetite/sleep disturbances and medically unexplained symptoms.

Additional analysis in the mother sample evidence an association between PPD and the

Table 4. Comparison between parents who desired the pregnancy and parents who not desire it.

\begin{tabular}{lccc} 
& Desired preginancy & No desired preginancy & Tot. \\
Mothers & & & \\
NO PPD & 37 & 5 & 42 \\
PPD & 11 & 0 & 11 \\
Tot. & 48 & 5 & 53 \\
Fathers & & & \\
NO PPD & 38 & 2 & 40 \\
SUB PPD & 7 & 3 & 10 \\
PPD & 3 & 0 & 3 \\
Tot. & 48 & 5 & 53 \\
\hline
\end{tabular}

PPD, postpartum depression.

Table 5. Comparison between mothers who breastfeed their child and mothers who not breastfeed them.

\begin{tabular}{lccc} 
& Breastfeed & No breastfeed & Tot. \\
No postpartum depressed mothers & 42 & 0 & 42 \\
Postpartum depressed mothers & 10 & 1 & 11 \\
\hline Total & 52 & 1 & 53 \\
\hline
\end{tabular}


interruption/decision to interruption to breastfeed their child confirming that the breastfeeding is a phase related to maternal stress and depression. ${ }^{45-50}$

Some authors conclude that maternal depression could cause the breastfeeding interruption, ${ }^{46}$ and that difficulties in breastfeeding could be considered the manifestation of PPD and parenting distress. ${ }^{51,52}$ The study results and the literature evidence could lead up to a circular logic: depression causes a decreased lactation, the mother decides to interrupt the breastfeeding and it exposes her to a depression risk. In the other hand, psychological depressive vulnerability could lead the mother to decide to suspend the breastfeeding for her perceived incompetence to feed her child or for perceived hostility toward her child.

Study results have reported also an high percentage of paternal postpartum depression risk, as resulted in literature, and this evidence suggests a reflection about fathers and paternal role. Nowadays, men experience many difficulties in the transition to parenthood because they have to compare themselves with male and traditional models and have to coping with changes regarding working and social role. This comparison could provoke a upsetting of personal and paternal balance. $.33,54$

Nevertheless, paternal PPD has been studied systematically only in the recent years and its diagnosis remains difficult since it appears with a light or atypical symptomatology, in association with others psychological and behavioral disturbances. 55

Like the woman, also the man lives the pregnancy and the childbirth such as a phase of psychological restructuration and he confronts himself with his personal and family history. 56 But, unlike the woman, the man does not experience an emotional exchange with the child during the pregnancy or after the childbirth and he establish the relation in the two months after the childbirth. 57,58 They experience important changes and they have more difficulty to begin a good affective relationship with the child than the mothers that establish it after the childbirth. The new-fathers could live impotence before an harmonic and emphatic relation between mother and child, exclusion sentiment and jealousy toward wife, ${ }^{59}$ child and their relation. They could perceive that the child drains the mother and that his relation with the wife is irremediably changed: sharing the time and the sexuality became difficult or impossible and it could provoke insecurity, 60 tiredness and, together with new responsibility and prior psychological disturbance, depression status. ${ }^{6}$

Another risk factor of paternal PPD could be the absence of a good paternal model: Barclay and Lupton evidenced that a lot of new-fathers who lived emotional difficult after childbirth had lived a sad childhood characterized by the absence (real o subjective) of their father. ${ }^{62}$ In this way, these men did not know parenting competence and, when they became father, live fear, insecurity, turmoil that could lead them to PPD.

Especially for fathers at first time, the incomprehension of paternal rule could cause anxiety and it is a risk factor for PPD. 60

Cox, Martins and Gaffan evidenced that the most important PPD risk factor is the history of a prior depression. ${ }^{63,64}$ Furthermore, the paternal PPD is more frequent in father who did not desire the pregnancy and who did not accept psychologically and physically the newborn. 65

The study results confirm this association between the desire of pregnancy absence and paternal PPD.

Unlike the mothers, the fathers who did not desire a child show more PPD vulnerability and more difficult in the psychological reorganization than fathers who have desired their child and who appear more ready to accept a new component. $66-68$

About our third aim, regarding the correlation between maternal and paternal psychological status, study results confirm literature evidence reporting that paternal risk of depression is lower than maternal one. ${ }^{8}$

Although the literature considers the maternal PPD such as predictive factor for paternal PDD in our study there are no correlations between maternal and paternal PPD. ${ }^{12,69}$

We believe that it could not due only to different onset period but also to different clinical manifestations of syndrome between genders. In the transition to parenthood new fathers and mothers experience different difficulties.

The correlations between maternal and paternal parenting distress indicate an evident concordance between experience of transition to parenthood that it was been perceived like a stressor.

Besides, it is not possible to suggest a certain direction of the relationship between paternal and maternal distress and we can speculate the influence of one partner on psychological status of the other one. It is more true for mood aspects, which are more subject to contagion than cognitive ones, especially during a developmental crisis like a birth of a child, as described in the introduction.

The absence of correlation between PPD and parenting distress could be also explained with methodological way. Indeed, EPDS and PSI-SF appeared moderately sensitive to a screening of the risk of postpartum depression and the risk of parenting distress during transition to parenthood.

\section{Study limitations}

A first study limit is the lack of prenatal depression assessment and a longitudinal study which should allow to deeply understand the clinical nature and course of maternal and paternal distress during transition to parenthood, especially for paternal depression which could onset later than maternal one.

An another limit concerns the use of selfreports which could be replaced or combined with clinical interviews more suitable in a clinical approach.

Furthermore, it could be prudent consider the role of other psychosocial variables on postpartum depression like social support perception, global psychological distress of partners, marital satisfaction, etc.

Finally, further researches could study the role of paternal and maternal postpartum depression on child development.

\section{Conclusions}

Transition to parenthood requires a reorganization of family functioning, the childbirth modify the family routine in terms of behaviors and psychological status.

Changes concerning couple relation and social role, physical and hormonal alteration, expectations on parenting role and child features emerged. During this critical life event, some of couples of parents experience an high vulnerability and refer significant distress levels. Mood disturbances and parenting stress in postpartum period represent high risks of parents and children well-being. Dysfunctional parenting has been assumed as an important risk factor in the development of psychological disturbances in adulthood and several studies have reported a significant correlation between maternal PPD and altered cognitive/ affective child development. ${ }^{16}$ Only a complex, clinical and multidisciplinary approach could deeply support the transition to parenthood and study results could be considered only a guidance in the assessment of psychopathologic disturbances.Furthermore, poor attention has been paid to the mood disturbance of fathers and to the association between depression and anxiety. To identify characteristics of parental PPD and distress using screening scales is a good way to overcome some difficulties and prevent family distress.

Recognizing and including the father as a participant in the childbirth process is an important task for midwives in order to promote emotional involvement between father and baby. 70

According to The World Health Organization, 71 depression can be reliably diagnosed and treated in primary care settings. Primary health care was defined by the WHO as an essential health care based on practical, scientifically sound and socially acceptable methods and technology, made universally available to 
individuals and families in the community. 72 Indeed, it has been demonstrated that mothers with postnatal depression had an increased utilization of health and social care services, resulting in higher economic costs.

The theme of postpartum distress treatment could be deepened to prevent and care major affective disorder in parents and developmental disturbances in children. For these reasons, we believe that pediatricians are essential figures to identify prematurely these risk conditions and promote childhood and parenting well-being and that they represent an important member of the multidisciplinary team for the child, parents and family care.

\section{References}

1. Condon JT, Boyce P, Corkindale CJ. The first-time fathers study: a prospective study of the mental health and wellbeing of men during the transition to parenthood. Aust N Z J Psychiatry 2004;38:56-64.

2. Monti F, Agostani F. La depressione postanale. Roma: Carocci; 2006.

3. AA.VV. DSM-5 Manuale diagnostico e statistico dei disturbi mentali. Milano: Raffaello Cortina Editore; 2014.

4. Caretti V, Ragonese N, Crisafi C, eds. La depressione perinatale. Aspetti clinici e di ricerca sulla genitorialità a rischio. Roma: Giovanni Fioriti Editore; 2013.

5. Lanes A, Kuk JL, Tamin H. Prevalence and characteristics of postpartum depression symptomatology among Canadian women: a cross-sectional study. BMC Public Health 2011;11:302.

6. Nagy E, Molnar P, Pal A, Orvos H. Prevalence rates and socioeconomic characteristics of post-partum depression in Hungary. Psychiatry Res 2011;185:113-20.

7. Letourneau N, Duffett-Legger L, Dennis L, et al. Identifying the support needs of fathers affected by post-partum depression: a pilot study. J Psychiatr Ment Health Nurs 2011;18:41-7.

8. Escribà-Aguir V, Artazcoz L. Gender differences in postpartum depression: a longitudinal cohort study. J Epidemiol Community Health 2011;65:320-6.

9. Ramchandani P, Stein A, Evans J, 0'Connor T. Paternal depression in the postata period and child development: a prospective population study. Lancet 2005;365:2201-5.

10. Kim P, Swain JE. Sad dads: paternal postpartum depression. Psychiatry 2007;4:3547.

11. Ramchandani P, Stein A, O'Connor TG, et al. Depression in men in the postnatal period and later child psychopathology: a population cohort study. J Am Acad Child
Adolesc Psychiatry 2008;47:390-8.

12. Goodman JH. Paternal postpartum depression, its relationship to maternal postpartum depression, and implications for family health. J Adv Nurs 2004;45:26-35.

13. Schumacher M, Zubaran C, White G. Bringing birth-related paternal depression to the fore. Women Birth 2008;21:65-70.

14. Winkler D, Pjrek E, Heiden A. Gender differences in the psychopathology of depressed inpatients. Eur Arch Psychiatry Clin Neurosci 2004;254:209-14.

15. Harvey I, McGrath G. Psychiatric morbidity in spouses of women admitted to a mother and baby unit. Br J Psychiatry 1988;152: 506-10.

16. Grussu P, Quatraro RM. Depressione materna del dopo parto e salute psicologica del bambino: contributi sperimentali e analisi critica della letteratura. Psicologia Clinica dello Sviluppo 2006;1:187-208.

17. Di Blasio P. Effetto dell'expressive writing sulla depressione post partum e sullo stress materno. In: Caretti V, Ragonese N, Crisafi C, eds. La depressione perinatale. Aspetti clinici e di ricerca sulla genitorialità a rischio. Roma: Giovanni Fioriti Editore; 2013.

18. Abidin, R. Parenting Stress Index: professional manual, 3rd edn. Odessa: Psychological Assessment Resources; 1995.

19. Guarino A, Di Blasio P, D’Alessio M, et al. Manuale PSI. Parent Stress Index. Firenze: Giunti O.S.; 2008.

20. Romito P, Saurel-Cubizzoles MJ. I costi della maternità nella vita delle donne. Polis 1997;11:67-88.

21. Istituto Nazionale di Statistica (ISTAT). Conciliare lavoro e famiglia. Una sfida quotidiana. Roma: Istat produzione libraria e centro stampa; 2008.

22. Gelfand DM, Teti DM, Radin Fox CE. Sources of parenting stress for depressed and nondepressed mothers of infants. J Clin Child Psychology 1992;21:262-72.

23. Beck CT. Predictors of postpartum depression: an update. Nurs Res 2001;50:275-85.

24. Field T. Postpartum depression effects on early interactions, parenting, and safety practices: a review. Infant Behav Dev 2010;33:1-6.

25. Store AN. The esperience-depedent maturation of a regulatory system in the orbital prefrontal cortex and the origin of developmental psychopathology. Dev Psychopathol 1996;8:59-87.

26. Lewis M, Granic I, eds. The self-organisation of the rigth brain and the neurobiology of emotional development. In: Emotion, development, and self-organisation, dynamic systems approaches to emotional development. Cambridge: Cambridge University Press; 2000.
27. Milgrom J, Ericksen J, McCarthy R, Gemmill AW. Stressfull impact of depression nearly mother-infant relations. Stress Healt 2006;22:229-38.

28. Logsdon MC, Wisner KL, Pinto-Foltz MD.. The impact of postpartum depression on mothering. J Obstet Gynecol Neonatal Nurs 2006;35:652-8.

29. Paulson JF, Dauber S, Leiferman JA. Individual and combined effects of postpartum depression in mothers and fathers on parenting behaviour. Pediatrics 2006;118:659-68.

30. Taaffe McLearn K, Minkovitz CC, Strobino $\mathrm{DM}$, et al. The timing of maternal depressive simptoms and mothers' parenting practices with young children: implications for pediatrics practice. Pediatrics. 2006;118:174-82.

31. Monti F, Agostini F, Martini A. Il silenzio degli affetti: la depressione post partum e il bambino. Clinica e ricerca. Contrappunto 2004;34:61-87.

32. Rigetti PL, Casadei D. Il sostegno psicologico in gravidanza. Roma: Ed. Scientifiche Ma.Gi. Collana Forma Mentis; 2006.

33. Della Vedova AM, Cristini C, eds. La promozione della salute psichica perinatale. Roma: Edizioni Carocci; 2011.

34. Mäntymaa M, Puura K, Luoma I, et al. Infant-mother interaction as a predictor of child's chronic health problems. Child Care Health Dev 2003;29:181-91.

35. Ammaniti M, Cimino S, Trentini C. Quando le madri non sono felici. La depressione post-partum. Roma: Il Pensiero Scientifico Editore; 2007.

36. Halligan SL, Murray L, Martins C, Cooper PJ. Maternal depression and psychiatric outcomes in adolescent offspring: a 13year longitudinal study. J Affect Disord 2007;97:145-54.

37. Sohr-Preston SL, Scaramella LV. Implications of timing of maternal depressive symptoms for early cognitive an language development. Clin Child Fam Psychol Rev 2006;9:65-83.

38. Lyons-Ruth K, Tambelli R, Zaccagnini C. Il trauma latente nel dialogo relazionale dell'infanzia. Roma: Borla; 2012.

39. Wu YP, Seling JP, Roberts MC, Steele RG. Trajectories of postpartum maternal depressive symptoms and children's social skills. J Child Fam Stud 2011;4:414-23.

40. Cox JL, Holden JM, Sagowsky R. Detection of the post natal depression: development of the 10-item Edimburgh Post natal depression scale. Br J Psychiatry 1987;150: 782-6.

41. Benvenuti P, Ferrara M, Niccolai C, et al. The Edinburgh Postnatal Depression Scale: validation for an Italian sample. J Affect Disord 1999;53:137-41. 
42. Matthey S, Barnett B, Kavanagh DJ, Howie P. Validation of the Edimburgh Postnatal Depression scale for man and comparison of item endorsement with their partners. J Affect Disord 2001;64:175-84.

43. World Health Organization. What is depression? 2008. Available from: http://www.who.int/mentalhealth/management/depression/definition/en.

44. Stern D. Motherhood constellation: a unified view of parent-infant psychotherapy, New York: Basic Book; 1995.

45. Lokuge S, Frey BN, Foster JA, et al. Depression in women: windows of vulnerability and new insights into the link between estrogen and serotonin. J Clin Psychiatry 2011:e1563-9.

46. Kendall-Tackett KA. Depression in new mothers. Binghanton. New York: Haworth; 2005.

47. Grajeda R, Perez-Escamilla R. Stress during labor and delivery is associated with delayed onset of lactation among guatemalan women. J Nutr 2002;132:305560.

48. Galler JR, Harrison RH, Biggs MA, et al. Maternal moods predict breastfeeding in Barbados. J Dev Behav Pediat 1999;20:807.

49. Misri S, Sinclair DA, Kuan AJ. Breasfeeding and postpartum depression: is there a relationship? Can J Psichiatr 1997;42:1061-5.

50. Bick DE, MacArthur C, Lancashire RJ. What influences the uptake and the early cessation of breastfeeding? Midwifery 1998;14:242-7.

51. Dennis CL, McQueen K. The relationship between infant-feeding outcomes and postpartum depression: a qualitative systematic review. Pediatrics 2009;123:73651.

52. Flores-Quijano ME, Córdova A, Contreras-
Ramírez V, et al. Risk for postpartum depression, breastfeeding practices, and mammary gland permeability. J Hum Lact 2008;24:50-7.

53. Garfield CF, Clark-Kauffman E, Davis MM. Fatherhood as a component of men's health. JAMA 2006;296:2365-8.

54. Zajczyk F, Ruspini E, Borlini B, Crosta F. Nuovi padri? Mutamenti della paternità in Italia e in Europa. Milano: Baldini Castoldi Dalai; 2008.

55. Baldoni F, Ceccarelli L. La depressione perinatale paterna. Una rassegna della ricerca clinica ed empirica. Infanzia e adolescenza. Rassegne 2010;9.

56. Scopesi A. L'uomo e la donna di fronte all'evento nascita del primo figlio. Bambino incompiuto 1990;1:75-83.

57. Edhborg M, Matthiesen AS, Lundh W, Widstrom AM. Some early indicators for depressive symptoms and bonding two month postpartum: a study of new mothers and fathers. Arch Womens Ment Health 2005;8:221-31.

58. Anderson AM. Factors influencing the father-infant relationship. J Fam Nurs 1996;2:306-24.

59. Rutter M, Caspi A, Fergusson D. Sex differences in developmental reading disability: new finding from 4 epidemiological studies. JAMA 2004;291:2007-12.

60. Ferketich SL, Mercer RT. Predictor of role competence for experienced and inexperienced fathers. Nurs Res 1995;44:89-95.

61. Goodman JH. Paternal postpartum depression, its relationship to maternal postpartum depression, and implications for family health. J Adv Nurs 2004;45:26-35.

62. Barclay L, Lupton D, eds. Biographies of fatherhood. In: Construction fatherhood. Newbury Park: SAGE Publication; 1999. pp 93-118.

63. Cox JL. Postnatal depression in fathers.
Lancet 2006;366:982-8.

64. Martins C, Gaffan EA. Effects of early maternal depression on patterns of infantmother attachment: a meta-analytic investigation. J Child Psychol Psychiatr 2000;41:737-46.

65. Nishimura A, Ohashi K. Risk factors of paternal depression in the early postnatal period in Japan. Nurs Health Sci 2010;12: 170-6.

66. Bouchard G, Boudreau J, Hébert R. Transition to parenthood and conjugal life. Comparison between planned and unplanned pregnancies. J Fam Issues 2006;27:1512-31.

67. Lawrence E, Rothman AD, Cobb RJ, et al. Marital satisfaction across the transition to parenthood. J Fam Psychol 2008;22:4150 .

68. Simpson JA, Rholes WS, Campbell L, et al. Adult attachment, the transition to parenthood, and depressive symptoms. J Pers Soc Psychol 2003;84:1172-87.

69. Pinheiro RT, Magalhães PV, Horta BL, et al. Is paternal postpartum depression associated with maternal postpartum depression? Population based-study in Brazil. Acta Psychiatr Scand 2006;113:230-2.

70. Brandão S, Figueiredo B. Fathers' emotional involvement with the neonate: impact of the umbilical cord cutting experience. J Adv Nurs 2012;68:2730-9.

71. World Health Organization. The World Health Report 2008. Primary Health Care. Report of the international conference on primary health care. Available from: http://www.who.int/whr/2008/whr08_en.pdf

72. World Health Organization. The World Health Organization, 1978. Primary health care. Report of the international conference on primary health care. Available from: http://whqlibdoc.who.int/publications/9241800011.pdf 